\title{
Stochastic modeling error reduction using Bayesian approach coupled with an adaptive Kriging based model
}

\author{
Ahmed Abouelyazied Abdallh*and Luc Dupré \\ Department of Electrical Energy, Systems and Automation, \\ Ghent University, Belgium
}

\section{Introduction}

Recently, the magnetic parameters of the magnetic core material inside an electromagnetic device (EMD), such as rotating electrical machines, have been retrieved using a coupled experimental-numerical electromagnetic inverse problem [1]. In these inverse problems, the measurements are interpreted using a forward model where the difference between the numerical model responses and the measurement quantities is iteratively minimized using a minimization algorithm. In practice, two major aspects can reduce the accuracy of the recovered solution of the inverse problem, specifically: measurement noise and inaccurate modeling. Measurement noise can be reduced to some extent by accurately performing the measurements. On the other hand, modeling errors basically originate from two main sources: the uncertain 'geometrical' model parameters and the way of modeling the physical phenomena of the EMD.

The effect of the uncertain geometrical model parameters on the solution of the inverse problem has been extensively investigated by the authors in several papers, see for example [2]. In these references, the EMD models are assumed to be perfect, i.e. all physical phenomena are modeled, or in other words, the EMD models exactly simulate the reality. To this end, the EMD needs to be modeled using a very complex numerical model, e.g. 3D finite element (FE) model with a very fine mesh discretization level and including very detailed material models. However, these 'fine' forward models are computationally expensive, and consequently the computational time of the inverse problem becomes incredible due to its iterative nature. Alternatively, simplified fast but less accurate 'coarse' models can be used instead of the fine models. In this case, a large recovery error in the inverse problem solution is expected due to the modeling error originating from the simplification in the used forward model. In order to compensate this modeling error, the Bayesian approximation error approach can be used by modifying the objective function to be minimized with the misfit between fine and coarse forward model responses. However, there are some drawbacks in the application of this technique such as the considerable computational time and the assumption of having a stochastic modeling error with a uniform distribution.

In this paper, we present a robust methodology in order to overcome these drawbacks. The main goal of this paper is to decrease the modeling error using the Bayesian approximation error approach coupled with an efficient, fast with a reasonable level of accuracy, Kriging based model. The proposed methodology comprises two steps: the first step is the simplification of the fine model using an adaptive Kriging based model, and the second step is the implementation of the Bayesian approximation error approach for compensating the modeling error.

\footnotetext{
*A. Abdallh is a postdoctoral researcher of the BOF "Bijzonder Onderzoeksfonds" of Ghent University. Corresponding author e-mail: ahmed.abdallh@ieee.org
} 
The proposed methodology is validated experimentally for identifying the material parameters of a real EMD, i.e. an EI electromagnetic core inductor, by solving an inverse problem starting from real experimental data.

The methodology is presented in section 2. In section 2.1, the Bayesian approximation error approach is presented followed by a brief summary of the adaptive Kriging based model coupled with the cross-validation technique in section 2.2. As a real case study, the proposed methodology is validated experimentally on an EI electromagnetic core inductor in section 3. The obtained results are discussed in section 4. Finally, the conclusions are drawn in section 5.

\section{Methodology}

In this section, we briefly explain the application of the Bayesian approximation error approach for modeling error reduction in the solution of the inverse problem. In order to reduce the computational time as well as improve the accuracy of the inverse problem solution, the adaptive Kriging modeling technique is proposed.

\subsection{The Bayesian approximation error approach}

Electromagnetic devices can be modeled using different degrees of freedom. The three-dimensional finite element (3DFE) models with very fine mesh discretization are often utilized for accurate simulation of EMDs. Instead, less accurate but fast coarse models can be used as a rough approximation of the reality. In order to reduce the recovery error in the inverse problem due to the implementation of the coarse model instead of the fine model, the Bayesian approximation error approach has been successfully used [3].

The Bayesian approximation error approach is a stochastic technique based on a priori modification of the objective function to be minimized by the misfit between the fine and coarse model. Traditionally, the objective function to be minimized in the inverse problem framework is the quadratic residuals between the experimental observations of the magnetic system $\mathbf{W} \in \mathbb{R}^{K \times 1}$ and the modeled ones $\Phi \in \mathbb{R}^{K \times 1}$, with $\mathbf{u}$ and $K$ being the unknown parameters to be estimated and the total number of discrete experimental observations, respectively. In other words, the functional

$$
O F(\mathbf{u})=[\mathbf{\Phi}(\mathbf{u})-\mathbf{W}]^{T}[\mathbf{\Phi}(\mathbf{u})-\mathbf{W}]
$$

needs to be minimized:

$$
\tilde{\mathbf{u}}=\arg \min _{\mathbf{u}} O F(\mathbf{u})
$$

with $\tilde{\mathbf{u}}$ being the recovered values of the unknown model parameters. In practice, $\tilde{\mathbf{u}}$ is not necessarily equal to the actual model parameters $\mathbf{u}^{*}$, due to the presence of the measurement noise $\mathbf{e}_{n}$ and modeling error $\mathbf{e}_{m}$, i.e. $\mathbf{W}=\boldsymbol{\Phi}\left(\mathbf{u}^{*}\right)+\mathbf{e}_{n}+\mathbf{e}_{m}$.

Assume that the fine model, which is used for modeling the EMD, simulates perfectly the physics of the reality, i.e. $\mathbf{W}=\boldsymbol{\Phi}_{f}\left(\mathbf{u}^{*}\right)+\mathbf{e}_{n}$. Alternatively, one may use a coarse model for modeling the EMD with an expected discrepancy between the fine and the coarse model responses, which is referred as modeling error, i.e. $\mathbf{W}=\boldsymbol{\Phi}_{c}\left(\mathbf{u}^{*}\right)+\mathbf{e}_{n}+\mathbf{e}_{m}$. Since the exact value of the parameter $\mathbf{u}$ is not known in advance, the modeling error is calculated as the misfit between the fine and the coarse model responses using $Z$ hypothetical values of the unknown model parameters, with $\widehat{\mathbf{u}}_{z}, z=1, \cdots, Z$ being a hypothetical value. These $Z$ hypothetical values are chosen in such a way that they are random and cover the domain defined by the lower and upper bounds of these parameters.

The error between the fine and coarse forward models, at each model observation $k$ and at each test value $\widehat{\mathbf{u}}_{z}$, can be represented by:

$$
e_{m, k}\left(\widehat{\mathbf{u}}_{z}\right)=\Phi_{f, k}\left(\widehat{\mathbf{u}}_{z}\right)-\Phi_{c, k}\left(\widehat{\mathbf{u}}_{z}\right), \quad(k=1, \cdots, K), \quad(z=1, \cdots, Z)
$$


with $K$ and $Z$ being the total number of discrete model observations and the total number of the assumed hypothetical values of the unknown model parameters, respectively. By performing $Z$ coarse and fine forward model computations, and assuming that the modeling error at each model observation $k\left(e_{m, k}\right)$ follows the normal distribution, i.e. $\left(e_{m, k} \sim\right.$ $\left.\mathcal{N}\left(\mu_{m, k}, \sigma_{m, k}^{2}\right)\right)$, one may calculate the mean modeling error and its variance, $\mu_{m, k}$ and $\sigma_{m, k}^{2}$, respectively.

$$
\mu_{m, k}=\frac{1}{Z} \sum_{z=1}^{Z} e_{m, k}\left(\widehat{\mathbf{u}}_{z}\right), \quad \sigma_{m, k}^{2}=\frac{1}{Z} \sum_{z=1}^{Z}\left(e_{m, k}\left(\widehat{\mathbf{u}}_{z}\right)-\mu_{m, k}\right)^{2}
$$

Based on the calculated $\mu_{m, k}$ and $\sigma_{m, k}^{2}$, the approximate overall probability distribution function (PDF), at each model observation $k$, can be written as:

$$
f\left(e_{m, k}\right)=\frac{1}{\sqrt{2 \pi \sigma_{m, k}^{2}}} \cdot \exp \left(-\left(e_{m, k}-\mu_{m, k}\right)^{2} /\left(2 \sigma_{m, k}^{2}\right)\right)
$$

The vector representations of the modeling mean error and its variance at all model observations $K$ are:

$$
\overline{\boldsymbol{\mu}}_{m}=\left[\mu_{m, 1}, \mu_{m, 2}, \cdots, \mu_{m, K}\right]^{T}, \overline{\boldsymbol{\sigma}}_{m}^{2}=\left[\sigma_{m, 1}^{2}, \sigma_{m, 2}^{2}, \cdots, \sigma_{m, K}^{2}\right]^{T}
$$

Since the modeling error $\mathbf{e}_{m}$ is assumed uncorrelated, i.e. $e_{m, i}$ does not depend on $e_{m, j}(i, j=1, \cdots, K, i \neq j)$, the covariance matrix of the modeling error $\left(\overline{\boldsymbol{\Sigma}}_{m}^{2} \in \mathbb{R}^{K \times K}\right)$ can be written as: $\overline{\boldsymbol{\Sigma}}_{m}^{2}=\operatorname{diag}\left(\sigma_{m, 1}^{2}, \sigma_{m, 2}^{2}, \cdots, \sigma_{m, K}^{2}\right)$. The mean and the covariance of the modeling error are used later for compensating the modeling error.

On the other hand, due to the random nature of the measurement noise $\mathbf{e}_{n}$, it is assumed to be normally white distributed with zero mean $\left(\bar{\mu}_{n, k}=0\right)$ and a variance of $\bar{\sigma}_{n, k}^{2}$, i.e. $\left(\mathbf{e}_{n, k} \sim \mathcal{N}\left(0, \bar{\sigma}_{n, k}^{2}\right)\right)$ [3]. Similarly,

$$
\overline{\boldsymbol{\mu}}_{n}=\left[\mu_{n, 1}, \mu_{n, 2}, \cdots, \mu_{n, K}\right]^{T}=\mathbf{0}, \overline{\boldsymbol{\sigma}}_{n}^{2}=\left[\sigma_{n, 1}^{2}, \sigma_{n, 2}^{2}, \cdots, \sigma_{n, K}^{2}\right]^{T}
$$

Again, since the measurement noise is assumed uncorrelated, i.e. $e_{n, i}$ does not depend on $e_{n, j}(i, j=1, \cdots, K, i \neq j)$, the covariance matrix of the measurement noise $\left(\bar{\Sigma}_{n}^{2} \in \mathbb{R}^{K \times K}\right)$ can be written as: $\bar{\Sigma}_{n}^{2}=\operatorname{diag}\left(\sigma_{n, 1}^{2}, \sigma_{n, 2}^{2}, \cdots, \sigma_{n, K}^{2}\right)$.

In the Bayesian framework, the identification problem is seen as a statistical inference problem, in which the measurements and the modeled responses are assumed to be random [4]. In the Bayesian approximation error approach, the forward model can be rewritten as: $\mathbf{W}=\boldsymbol{\Phi}_{c}\left(\mathbf{u}^{*}\right)+\mathbf{e}_{n}+\mathbf{e}_{m}$. Due to the Gaussian distribution of both the measurement noise and modeling error, the overall error $\mathbf{e}$ is also Gaussian distributed.

In the well known Bayes' formula, the posterior probability density function of the measurements $\mathbf{W}$ given the unknown model parameters $\mathbf{u}$ ' $P(\mathbf{u} \mid \mathbf{W})$ ' is given by [5]:

$$
P(\mathbf{u} \mid \mathbf{W})=\frac{P(\mathbf{u}) P(\mathbf{W} \mid \mathbf{u})}{P(\mathbf{W})}
$$

which can be written in a non-normalized form:

$$
P(\mathbf{u} \mid \mathbf{W}) \propto P(\mathbf{u}) P(\mathbf{W} \mid \mathbf{u})
$$

with $P(\mathbf{u})$ being the prior probability density function of the unknown model parameters.

Since no information is given for the $P(\mathbf{u})$ in our application, the unknown model parameters are assumed to follow the uniform distribution between lower and upper bounds; $\mathbf{u}_{L B}$ and $\mathbf{u}_{U B}$, respectively:

$$
P(\mathbf{u})=\frac{1}{\mathbf{u}_{U B}-\mathbf{u}_{L B}}, \quad \mathbf{u} \in\left[\mathbf{u}_{L B}, \mathbf{u}_{U B}\right]
$$

$\mathbf{u}_{L B}$ and $\mathbf{u}_{U B}$ can be known from the reasonable physical representation of $\mathbf{u}$. 
Assuming neither $\mathbf{e}_{n}$ nor $\mathbf{e}_{m}$ depend on the unknown model parameters $\mathbf{u}$, the likelihood density function of the measurements $\mathbf{W}$ given the unknown model parameters $\mathbf{u}$ can be written as [5]:

$$
\begin{aligned}
P(\mathbf{W} \mid \mathbf{u})= & \frac{1}{(2 \pi)^{K / 2} \prod_{k=1}^{K} \sigma_{n, k} \cdot \sigma_{m, k}} \\
& \cdot \exp \left(-\frac{1}{2}\left[\left[\mathbf{W}-\mathbf{\Phi}(\mathbf{u})-\overline{\boldsymbol{\mu}}_{n}-\overline{\boldsymbol{\mu}}_{m}\right]^{T}\left(\overline{\boldsymbol{\Sigma}}_{n}^{2}-\overline{\boldsymbol{\Sigma}}_{m}^{2}\right)^{-1}\left[\mathbf{W}-\boldsymbol{\Phi}(\mathbf{u})-\overline{\boldsymbol{\mu}}_{n}-\overline{\boldsymbol{\mu}}_{m}\right]\right]\right)
\end{aligned}
$$

Therefore, in order to solve this inverse problem, the maximum a posteriori ( $M A P$ ) estimates is used, in which the $M A P$ of the unknown model parameters $\mathbf{u}$ is given by:

$$
\mathbf{u}_{\mathrm{MAP}}=\arg \max _{\mathbf{u}} P(\mathbf{u} \mid \mathbf{W})
$$

Substituting (9) and (11) in (12), and $\left(\overline{\boldsymbol{\mu}}_{n}=0\right)$ :

$$
\begin{aligned}
\mathbf{u}_{\mathrm{MAP}, \mathrm{Trad}} & =\arg \max _{\mathbf{u}} P(\mathbf{W} \mid \mathbf{u}) \\
& =\arg \max _{\mathbf{u}}\left\{\exp \left(-\frac{1}{2}\left[\left[\mathbf{W}-\boldsymbol{\Phi}(\mathbf{u})-\overline{\boldsymbol{\mu}}_{m}\right]^{T}\left(\overline{\boldsymbol{\Sigma}}_{n}^{2}+\overline{\boldsymbol{\Sigma}}_{m}^{2}\right)^{-1}\left[\mathbf{W}-\boldsymbol{\Phi}(\mathbf{u})-\overline{\boldsymbol{\mu}}_{m}\right]\right]\right)\right\} \\
& =\arg \min _{\mathbf{u}}\left\{\left[\mathbf{W}-\mathbf{\Phi}(\mathbf{u})-\overline{\boldsymbol{\mu}}_{m}\right]^{T}\left(\overline{\boldsymbol{\Sigma}}_{n}^{2}+\overline{\boldsymbol{\Sigma}}_{m}^{2}\right)^{-1}\left[\mathbf{W}-\boldsymbol{\Phi}(\mathbf{u})-\overline{\boldsymbol{\mu}}_{m}\right]\right\} \\
& =\arg \min _{\mathbf{u}}\left\|\mathbf{L}_{n+m}\left(\mathbf{W}-\boldsymbol{\Phi}(\mathbf{u})-\overline{\boldsymbol{\mu}}_{m}\right)\right\|^{2}
\end{aligned}
$$

with $\mathbf{L}_{n+m}$ being the Cholesky factor of the covariance of the overall error, i.e. $\left(\overline{\boldsymbol{\Sigma}}_{n}^{2}+\overline{\boldsymbol{\Sigma}}_{m}^{2}\right)^{-1}=\mathbf{L}_{n+m}^{T} \mathbf{L}_{n+m}$. $\overline{\boldsymbol{\Sigma}}_{n}^{2}$ and $\overline{\boldsymbol{\Sigma}}_{m}^{2}$ are the covariance matrix of the measurement noise and modeling error, respectively. $\overline{\boldsymbol{\mu}}_{m}$ is the vector of the mean values of the modeling error. Solution of (13) is the recovered model parameter ( $\mathbf{u}_{\mathrm{MAP}}$, Compensated $\equiv \widetilde{\mathbf{u}}$ ) using the inverse problem in the Bayesian approximation error approach, in which the modeling error is compensated. For more information concerning the Bayesian approximation error approach, see [4], [6].

Although the Bayesian approximation error approach is successfully applied for reducing the effect of the modeling error when the inverse problem is solved, we discovered some difficulties. In [3], the Bayesian technique is applied to reduce the modeling error in the inverse problem solution when a 2D-FE or an analytical model is used instead of a 3DFE model. These coarse models are rather computationally expensive. Moreover, the accuracy of the coarse model was fixed 'to some extent', i.e. can not be largely improved. In other words, the capability of enhancing the accuracy of the coarse models was not considered in [3]. Furthermore, the objective function is adapted by the misfit between the fine and the coarse model responses, which assumed to follow the normal distribution. This assumption rarely occurred. The modeling error may or may not have a normal distribution. In fact, the distribution of the modeling error should be tested before applying the Bayesian approximation error approach. If the error can be approximated by the normal distribution, then the Bayesian approximation error approach can be used accurately. Nevertheless, a modeling error still appears.

Therefore, definitely there is a need to modify the error reduction technique using the Bayesian approximation error approach where the coarse model should be faster, its accuracy can be adapted, and the modeling error between the fine and the coarse model should follow the normal distribution. For these reasons, we propose the use of the adaptive Kriging based model.

\subsection{An adaptive Kriging based model}

Kriging models are widely used for modeling EMDs [7]. Suppose that the aim is to simplify the fine forward model: $\mathbf{Y}(\mathbf{u})=\Phi_{f}(\mathbf{u})$ using a Kriging model, where $\mathbf{Y}$ is the forward model response and $\mathbf{u}$ is the vector of the unknown 
parameters that needs to be identified using the inverse problem. The most common form of Kriging models is $\breve{\mathbf{Y}}(\mathbf{u})=$ $\boldsymbol{\beta}+\mathbf{E}(\mathbf{u})$, where $\breve{\mathbf{Y}}(\mathbf{u})$ is a simplified response of the forward model, and $\boldsymbol{\beta}$ is a constant. $\mathbf{E}(\mathbf{u})$ is the vector of stochastic modeling error with zero mean and variance $\sigma^{2}$, i.e. both $\boldsymbol{\beta}$ and $\mathbf{E}(\mathbf{u})$ are the output of the implementation of the Kriging technique to the considered model response. In fact, it is crucial to assess the accuracy of the simplified model before using it in the inverse procedure. To this end, we use the cross-validation scheme which is a very fast model accuracy assessment technique [8].

Based on the calculated accuracy level, the Kriging model is adapted iteratively by increasing the number of the used data-set $N$ until satisfying a predefined value of the accuracy level $\epsilon$. The use of the adaptive Kriging based model as a coarse model coupled with the Bayesian approximation error approach offers three advantages. The Kriging based metamodels are computationally inexpensive compared to the physical-based models, i.e. 2D-FE or analytical models. In this paper, the accuracy of the Kriging coarse model is improved to ensure an acceptable accuracy level before being used in the inverse problem. Moreover, the modeling error due to the simplification of the fine model using the Kriging model has a stochastic nature and purely follows a normal distribution. These three-fold advantages may improve the accuracy of the inverse problem solution.

\section{Application: An EI electromagnetic core inductor}

In this section, the proposed methodology is validated experimentally for the magnetic material identification of an EI core electromagnetic inductor.

\subsection{Problem definition and inverse problem formulation}

In this application, the magnetic material properties of the EI electromagnetic core inductor, shown in Fig. 1, need to be recovered using a coupled experimental-numerical electromagnetic inverse problem. The value of the middle air gap $g_{1}$ is $0.85 \mathrm{~mm}$, however, the value of $g_{2}$ is kept zero in order to eliminate the modeling uncertainty caused by the uncertain value of $g_{2}$, see [2]. In order to prevent the vibration during the experimental work, the two yokes are fixed together by a mechanical clamp.

In order to recover the values of the unknown magnetic material parameters, an electromagnetic inverse problem needs to be solved by interpreting the well-defined experimental measurements with a forward model. Here, we assume that the values of all geometrical parameters are perfectly known, and there are no uncertain model parameters. This assumption is acceptable due to the small effect of the uncertainty in the value of $g_{1}$ when the value of $g_{2}$ is kept zero, see [2]. However, the only considered uncertainty is the modeling uncertainty which originates from the simplification of the forward model.

The inverse problem is solved starting from local induction measurements at position $P$ on the $I$-yoke. The local induction measurements are obtained as the peak values of the hysteresis loops corresponding to $K=14$ amplitudes of the sinusoidal excitation current. The quasi-static magnetic measurements are performed at $1 \mathrm{~Hz}$ for a sinusoidal current excitation, in order to have a negligible skin effect due to the presence of eddy currents in the magnetic core. The measurements are performed five times and the average values are used as the input for the inverse problem. Here, we assume that the measurements are noise-free.

The single-valued nonlinear constitutive characteristic (normal magnetizing characteristic), to be reconstructed by the inverse problem, of the magnetic material of the EI core inductor is a nonlinear relation between the magnetic induction $B$ and the magnetic field strength $H$ without introducing the hysteresis behavior. There are many formulas to approximate 


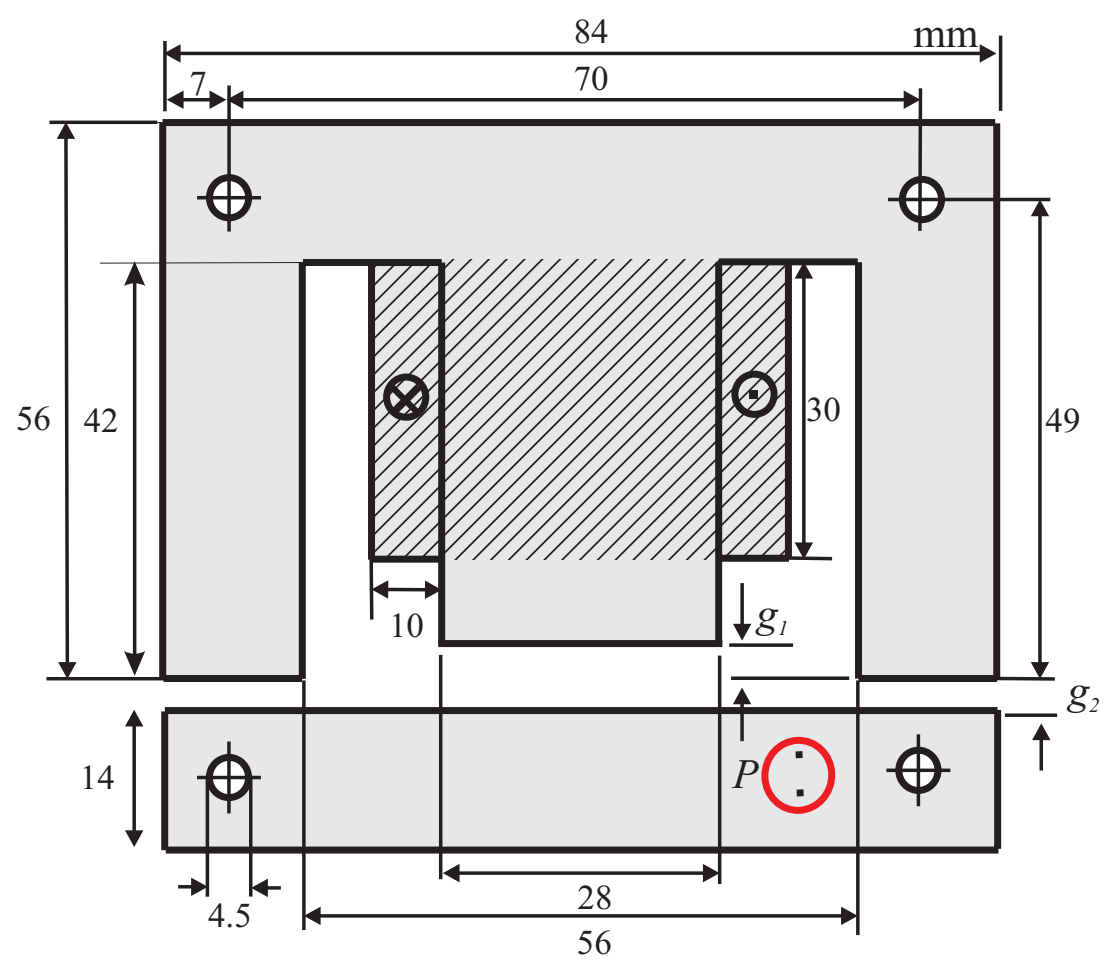

Figure 1: Schematic diagram of the studied EI electromagnetic core inductor.

the magnetization curve analytically [9]; one of them is a (non-full) power-series equation [10]:

$$
\frac{H}{H_{0}}=\left(\frac{B}{B_{0}}\right)+\left(\frac{B}{B_{0}}\right)^{\nu}
$$

with parameters $\mathbf{u}=\left[H_{0}(\mathrm{~A} / \mathrm{m}), B_{0}(\mathrm{~T}), \nu\right]$. The values of these parameters are unknown and need to be identified using an inverse approach. The magnetic reluctivity $v$ as a function of magnetic induction $B$ can be easily deduced from (14):

$$
v(B)=\frac{H_{0}}{B_{0}}\left(1+\left(\frac{B}{B_{0}}\right)^{\nu-1}\right) .
$$

The traditional objective function to be minimized is:

$$
\widetilde{\mathbf{u}}_{\mathrm{Trad}}=\arg \min _{\mathbf{u}}\left\|\mathbf{B}_{\mathrm{m}}-\mathbf{B}_{\mathrm{s}}(\mathbf{u})\right\|^{2}
$$

where $\mathbf{B}_{\mathrm{m}}$ and $\mathbf{B}_{\mathrm{s}}$ are the measured local magnetic induction at position $P$ and the corresponding simulated one, respectively. On the other hand, the modified objective function using the Bayesian approximation error approach is:

$$
\widetilde{\mathbf{u}}_{\text {Bayes }}=\arg \min _{\mathbf{u}}\left\|\mathbf{L}_{m}\left(\mathbf{B}_{\mathrm{m}}-\mathbf{B}_{\mathrm{s}}(\mathbf{u})-\overline{\boldsymbol{\mu}}_{m}\right)\right\|^{2}
$$

Here, the modeling error $\mathbf{e}_{m}$ is assumed uncorrelated. Therefore, the covariance matrix is diagonal, i.e. $\bar{\Sigma}_{m}^{2}=$ $\operatorname{diag}\left(\sigma_{m, 1}^{2}, \sigma_{m, 2}^{2}, \cdots, \sigma_{m, K}^{2}\right)$. In such a case, the Cholesky factorization is reduced by taking the square root. In (17), $\overline{\boldsymbol{\mu}}_{m}$ is calculated as mentioned in (6).

Moreover, it is worth mentioning that (16) and (17) are solved using the well-known least-square non-linear algorithm, i.e. Levenberg-Marquardt method with line search [11].

\subsection{EMD modeling}

Here, we build three models: a very fine model based on 3D-FE with very fine mesh discretizations, an analytical model based on the magnetic reluctance network theory (MRN), and an adaptive Kriging based model. 


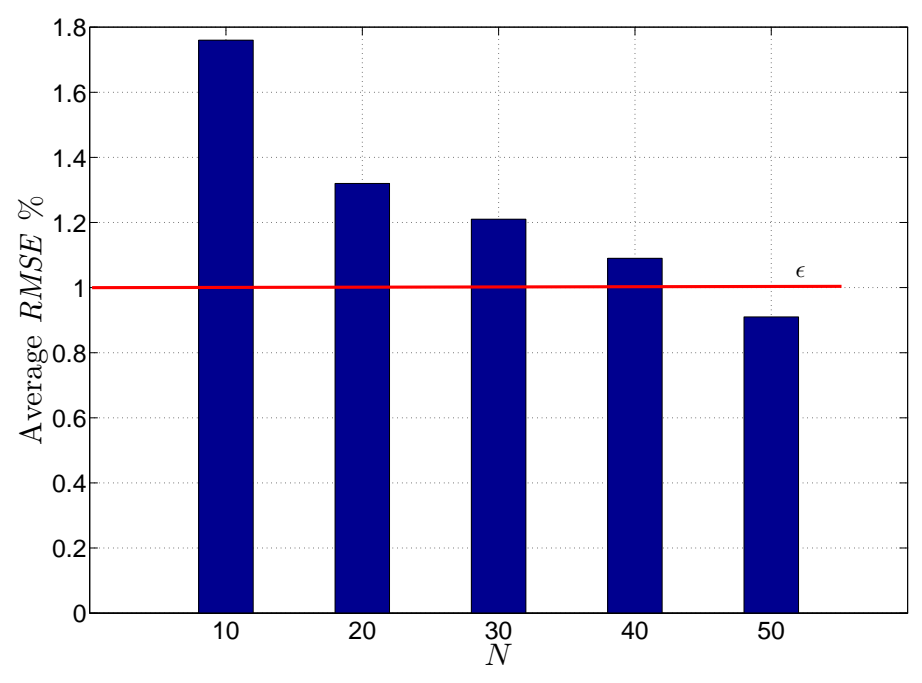

Figure 2: The average $R M S E$ values for different data-sets in the adaptive Kriging model. The value of the average $R M S E$ is calculated using the cross-validation technique.

For the adaptive Kriging based model, we initially use 10 data-set, i.e. $N=10$, and calculate the $R M S E$ using the cross-validation technique. The value of $N$ is iteratively increased with a step of 5 data-sets till reaching the stopping criterion, i.e. $\epsilon \leq 1 \%$. Fig. 2 shows the values of the $R M S E$ for only 5 Kriging modes built using different values of $N$. It is clear from this figure that the accuracy of the coarse model is adapted and improved with increasing $N$. The acceptable Kriging model for $(N=50)$ is used as a coarse model in the inverse problem combined with the Bayesian approximation error approach. The computational cost of calibrating the coarse model is approximately $N \times t_{\text {fine }}$, where $t_{\text {fine }}$ is the computational time of one forward computation of the fine model, see later Table 2.

It is worth mentioning that equation (16) is solved for the three models, however, (17) is solved only for the two 'relative' coarse computer models, i.e. MRN and Kriging based models, because no modeling error is considered in the fine model.

\subsection{Experimental validation}

The recovered magnetic material characteristics using the inverse problems are compared with the original normal magnetizing $B-H$ curve of the material, which is measured using the IEEE standard $393-1991$, see [12]. The original $B-H$ curve is fitted by equation (14), which results in the actual material parameter values $\mathbf{u}^{*}=[292.03(\mathrm{~A} / \mathrm{m}), 1.35(\mathrm{~T}), 11.99]$.

Different inverse problems are solved, with the assumption of $\mathbf{e}_{n}=0$, for each computer model. Then, the identified magnetic characteristics (single-valued $B-H$ curve) are compared. For the fine model, only one inverse problem is solved based on the traditional Bayesian approach (16). However, for the other two coarse models, four inverse problems are solved, two for each model. The first inverse problem is based on the traditional Bayesian approach (16), in which the modeling error is not compensated. While, the other inverse problem is based on the Bayesian approximation error approach (17), in which the modeling error is compensated.

Fig. 3 illustrates the solution of the five inverse problems compared to the original characteristics. It is clear from this figure that the application of the Bayesian approach for the Kriging model results in a better solution compared to the one based on the MRN. Table 1 shows the values of the recovery error for the three models. The recovery error is calculated using the formula presented in [3], which is based on the ratio between the recovered and the original $B$ - $H$ 


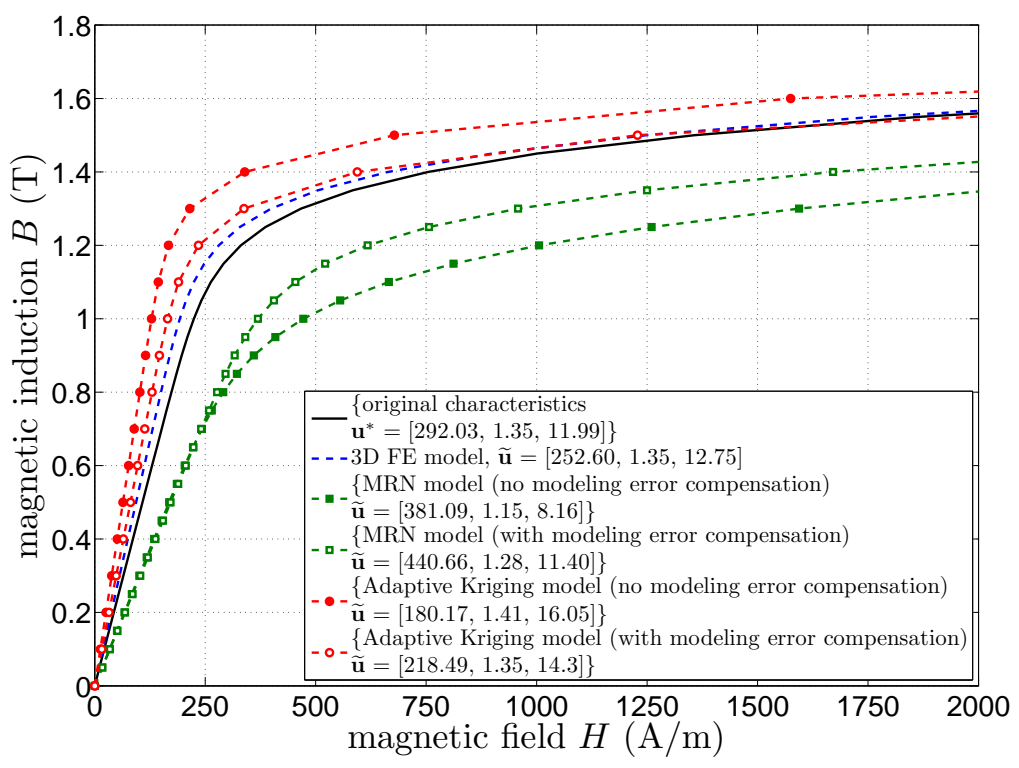

Figure 3: The recovered $B-H$ curve using the two inverse problems based on the traditional Bayesian approach "no modeling error compensation' and the Bayesian approximation error approach 'with modeling error compensation' for the two coarse models, and the recovered characteristics for the fine model, compared to the original characteristics.

Table 1: The values of the recovery error for the three models.

\begin{tabular}{|c|c|c|c|c|c|}
\hline Model & Fine model & MRN-model & MRN-model & Kriging model & Kriging model \\
\hline Compensation & - & no & yes & no & yes \\
\hline$R E \%$ & 0.0266 & 3.2304 & 1.5953 & 1.4813 & 0.0312 \\
\hline
\end{tabular}

curves, respectively.

The computational time required for solving the inverse problem based on the adaptive Kriging model is largely reduced compared the ones based on fine and MRN models. The main difference between the computational time of the inverse problems for the adaptive Kriging and the MRN model is that the modeling error is calculated when the Kriging model is being built. There is no need to perform extra computation to construct the stochastic modeling error. It is worth mentioning that the computational time for performing the cross-validation technique is negligible. Table 2 shows the computational time, in minutes, required for solving (16) and (17) for the MRN and the adaptive Kriging models compared to the time required for solving (16) for the fine model, assuming $Z=100$ and $N=50$ and the number of iterations equal to 100 .

Table 2: The computational time, in minutes, required for solving inverse problem based on the fine, MRN, and the adaptive Kriging models, assuming $Z=100, N=50$, and the number of iterations $=100$.

\begin{tabular}{|c|c|c|c|}
\hline Model & $\begin{array}{c}\text { One forward evaluation } \\
\text { time (min) }\end{array}$ & $\begin{array}{c}\text { Inverse problem time (min) } \\
\text { (without compensation) } \\
\text { "100 iterations" }\end{array}$ & $\begin{array}{c}\text { Inverse problem time (min) } \\
\text { (with compensation) } \\
\text { "100 iterations" }\end{array}$ \\
\hline Fine model & 10 & $4 \times 10^{3}$ & - \\
\hline MRN model & 0.1667 & 66.68 & $1.08 \times 10^{3}(Z=100)$ \\
\hline Kriging model & 0.002025 & 0.81 & $500.91(N=50)$ \\
\hline
\end{tabular}




\section{Discussion}

The proposed methodology has been developed to overcome some drawbacks in the previous work presented by the authors [3]. The Bayesian approximation error approach is coupled with a fast and relatively accurate coarse model based on a Kriging metamodel.

In practice, there are several techniques to reduce the modeling error. Two-level methods, such as space mapping technique [13], can reduce the modeling error by incorporating a coarse model with a fine one. Although the two-level techniques are worthy, the implementation of these techniques in the inverse problem requires a considerable computational time, because both fine and coarse models are solved in the iterative inverse approach. Moreover, a more advanced technique, i.e. the two-level refined direct method, was presented in [14] to reduce the modeling error when the finite element model with a coarse mesh is used instead of the fine one. Although the results presented in [14] are acceptable, the implementation of this technique requires advanced computations. Alternatively, the coupled Kriging-Bayesian approach, presented in this paper, offers a much easier and faster inverse scheme for reducing the modeling errors. Furthermore, the authors are convinced that the presented methodology is useful when dealing with high dimensional inverse problems, where the high computational cost of the forward problem is prohibitive.

\section{Conclusion}

In this paper, the recovery error in the inverse problem solution due to the simplification in the used forward model is reduced by implementing the Bayesian approach coupled with the stochastic Kriging model. The accuracy of the coarse model is adapted and improved a priori, using the cross-validation technique, to ensure an acceptable level of the model accuracy. The proposed methodology is validated experimentally for the magnetic material identification of an EI electromagnetic core inductor. Three computer models are used to model the EI core inductor. Beside the fine model, we use two relatively coarse models. The first coarse model is based on the theory of the magnetic reluctance network, while the other one is based on the adaptive Kriging metamodel. The main advantage of the proposed methodology is the high capability of reducing the effect of the modeling error in a relatively small computational time. The computational time is decreased approximately by about 50 percent with a better level of accuracy of the inverse problem. Finally, the presented scheme may be useful especially in the case of high dimensional inverse problems, where the light computational cost of the forward problem is necessarily needed.

\section{References}

[1] D. Ioan, M. Rebican, and A. Gasparics, " $B-H$ characteristic extraction using devices with non-uniform field," $C O M$ PEL, vol. 18, no. 3, 1999, pp. 469-481.

[2] A. Abdallh, G. Crevecoeur, and L. Dupré, "A priori experimental design for inverse identification of magnetic material properties of an electromagnetic device using uncertainty analysis," COMPEL, vol. 31, no. 3, 2012, pp. 972-984.

[3] A. Abdallh, G. Crevecoeur, and L. Dupré, "A Bayesian approach for the stochastic modeling error reduction of magnetic material identification of an electromagnetic device," Measurement Science and Technology, vol. 23, 2012, 035601(12pp). 
[4] A. Nissinen, L. Heikkinen, and L. Kaipio, "The Bayesian approximation error approach for electrical impedance tomography - experimental results," Measurement Science and Technology, vol. 19, 2008, 015501(9pp).

[5] J. Kaipio and E. Somersalo, Statistical and computational inverse problems (Applied Mathematical Sciences vol. 160). New York, USA: Springer, 2005.

[6] A. Nissinen, V. Kolehmainen, and J. Kaipio, "Compensation of errors due to incorrect model geometry in electrical impedance tomography," Journal of Physics: Conference Series, vol. 224, 2010, 012050.

[7] L. Wang and D. Lowther, "Selection of approximation models for electromagnetic device optimization," IEEE Transactions on Magnetics, vol. 42, no. 4, 2006, pp. 1227-1230.

[8] M. Meckesheimer and A. Booker, "Computationally inexpensive metamodel assessment strategies," AIAA Journal, vol. 40, no. 10, 2002, pp. 2053-2060.

[9] A. Abdallh, "An Inverse Problem Based Methodology with Uncertainty Analysis for the Identification of Magnetic Material Characteristics of Electromagnetic Devices," PhD Thesis, Ghent University, Belgium, 2012. Available online: https://biblio.ugent.be/publication/2125626.

[10] P. Sergeant, G. Crevecoeur, L. Dupré, and A. Van den Bossche, "Characterization and optimization of a permanent magnet synchronous machine," COMPEL, vol. 28, 2009, pp. 272-285.

[11] D. Marquardt, “An algorithm for least-squares estimation of nonlinear parameters," SIAM Journal on applied Mathematics, vol. 11, no. 2, pp. 431441, 1963.

[12] IEEE Standard 393-1991, "IEEE standard for test procedures for magnetic cores," Institute of Electrical and Electronics Engineers, 1992.

[13] A. Abdallh, G. Crevecoeur, P. Sergeant, and L. Dupré, "Magnetic material identification of a switched reluctance motor," International Journal of Applied Electromagnetics and Mechanics, vol. 37, pp. 35-49, 2011.

[14] G. Crevecoeur, A. Abdallh, I. Couckuyt, L. Dupré, and T. Dhaene, “Two-level refined direct optimization scheme using intermediate surrogate models for electromagnetic optimization of a switched reluctance motor," Engineering with Computers, vol. 28, no. 2, pp. 199-207, 2012. 